\title{
Correction to: Effects of periodontal treatment on carotid intima-media thickness in patients with lifestyle-related diseases: Japanese prospective multicentre observational study
}

\author{
Chieko Kudo $^{1}$ (D) Wee Soo Shin ${ }^{2} \cdot$ Nobuhiro Sasaki $^{3} \cdot \mathrm{Kazuo} \mathrm{Harai}^{4,5} \cdot \mathrm{Kai}^{\mathrm{Kato}}{ }^{6} \cdot \mathrm{Hiroaki}$ Seino $^{7} \cdot$ Eiji Goke $^{8}$. \\ Takemasa Fujino $^{9} \cdot$ Nobuichi Kuribayashi $^{10} \cdot$ Youko Onuki Pearce $^{11} \cdot$ Masato Taira $^{12} \cdot$ Ryoji Matsushima $^{13}$. \\ Masato Minabe ${ }^{1,14}$. Shogo Takashiba ${ }^{15}$. Periodontitis and Atherosclerosis Project-Tokyo and Chiba Consortiums
}

Published online: 20 February 2018

๑) The Society of The Nippon Dental University 2018

\section{Correction to: Odontology https://doi.org/10.1007/ s10266-017-0331-4}

Unfortunately, in Table 5 of the original article, the parameter in the 5th row was published incorrectly as "LDL-C (mg/dL)". The correct parameter should read as "HDL-C $(\mathrm{mg} / \mathrm{dL}) "$.
In addition, under the acknowledgment section, the following sentences were incorrectly published as Kouya Honda (Honda Medical Clinic, Tokyo) however it should read as Kouya Honda (Honda Medical Clinic, Nagasaki). Similarly, Yuichi Takakaze (Takakaze Dental Clinic, Tokyo) should read as Yuichi Takakaze (Takakaze Dental Clinic, Nagasaki).
The original article can be found online at https://doi.org/10.1007/ s10266-017-0331-4.

\section{Chieko Kudo}

kudo1974@gmail.com

1 Division of Periodontology, Department of Oral Function \& Restoration, Graduate School of Dentistry, Kanagawa Dental University, 82 Inaoka-cho, Yokosuka, Kanagawa 238-8580, Japan

2 Sekimachi Medical Clinic, 5-6-1 Sekimachikita, Nerima-ku, Tokyo 177-0051, Japan

3 Katsutadai Dental Clinic, 1-3-7 Katsutadai, Yachiyo-shi, Chiba 276-0024, Japan

4 Matsumoto Dental Office, 2-6-12-1F, Senzoku, Meguro-ku, Tokyo 152-0012, Japan

5 Present Address: Harai Dental Office, 2-20-17-1F Sangenjaya, Setagaya-ku, Tokyo 154-0024, Japan

6 Mejiro Medical-Road Dental Clinic, 2-5-27 Mejiro, Toshima-ku, Tokyo 171-0031, Japan

7 Tomiya Central Hospital, 2-1-6 Kamisakuragi, Tomiya-machi, Kurokawa-gun, Miyagi 981-3328, Japan
Goke Dental Clinic, 5-41-17 Higashioizumi, Nerima-ku, Tokyo 178-0063, Japan

9 Kyodo Dental Clinic, 1-32-2 Yoyogi, Shibuya-ku, Tokyo 151-0053, Japan

10 Misaki Internal Medicine Clinic, 6-44-9 Futawa Higashi, Funabashi-shi, Chiba 274-0805, Japan

11 Pearce Clinic, 7-20-5 Konakadai, Inage-ku Chiba-shi, Chiba 263-0043, Japan

12 Taira Clinic, 1-36-11 Katsutadai Yachiyo-shi, Chiba 276-0023, Japan

13 Matsushima Dental Clinic, 2-6-12-1F, Senzoku, Meguro-ku, Tokyo 152-0012, Japan

14 Bunkyo Do-ri Dental Clinic, 2-4-1, Anagawa, Inage-ku Chiba-shi, Chiba 263-0024, Japan

15 Department of Pathophysiology-Periodontal Science, Okayama University Graduate School of Medicine, Dentistry and Pharmaceutical Sciences, 2-5-1 Shikata-cho, Kita-ku, Okayama 700-8525, Japan 